\title{
Uso do tabaco entre funcionários de uma instituição de ensino superior
}

\author{
Ariane Regina Santos*, Karina Augustinho Bernardes*, Elaine Cristina Rocha Santos*, \\ Jéssica Steffany Miranda**, Silvio Fernando Guideti Marques***
}

*Acadêmicas do Curso de Enfermagem do Centro Universitário de Lins (UNILINS), Lins/SP, ** Docente do Curso de Enfermagem do Centro Universitário Católico Salesiano Auxilium de Lins (UNISALESIANO), Lins/SP, ***Docente do Curso de Enfermagem do Centro Universitário de Lins (UNILINS), Lins/SP

\section{Resumo}

O tabagismo é uma das maiores preocupaçóes da sociedade moderna, devido aos efeitos deletérios que acarreta na população. O conhecimento da prevalência do tabagismo é necessário para a realização de programas institucionais adequados que visem à diminuiçáo do número de fumantes. Pensando nesta situaçáo, o presente trabalho teve como objetivo avaliar a prevalência do tabagismo em funcionários da Fundação Paulista de Tecnologia e Educação (FPTE) de Lins/SP. Nesta análise foi aplicado um questionário a 149 funcionários e constatou-se a prevalência de tabagismo de 15,4\%. 74,5\% dos funcionários não eram fumantes e $10,1 \%$ foram classificados como ex-fumantes. A prevalência quanto ao gênero foi de $65,2 \%$ entre os homens e de 34,8\% entre as mulheres. A maioria fumava há mais de 9 anos $(87,0 \%)$ e iniciaram o tabagismo entre 16 e 18 anos de idade (43,4\%). A faixa etária de maior prevalência foi entre $45-49$ anos (30,5\%). Dos entrevistados classificados como não-fumantes (33,3\%), a maioria convive com fumantes no ambiente de trabalho (51,4\%). Embora a prevalência encontrada tenha sido menor que a de outros estudos realizados no Brasil, sugere-se a elaboração de medidas de conscientização sobre a prática tabágica visando à profilaxia do consumo de cigarros nesta população.

Palavras-chave: tabagismo, funcionários, prevalência, características.

\section{Abstract}

\section{Tobacco use among employees of an institution of higher education}

Smoking is a major concern in modern society due to the deleterious effects in the population. Knowledge of the prevalence of smoking is necessary to achieve adequate institutional programs aimed at reducing the number of smokers. Thinking about this situation, this study aimed to assess the prevalence of smoking among employees of Fundação Paulista de Tecnologia e Educação (FPTE) of Lins/SP. In this analysis, a questionnaire was administered to 149 employees and was found smoking prevalence of $15.4 \%$. $74.5 \%$ of employees were non-smokers and $10.1 \%$ were former smokers. The prevalence by gender smoking ratio was $65.2 \%$ among men and $34.8 \%$ among women. Most smoked for over 9 years $(87.0 \%)$ and began smoking between 16 and 18 years of age (43.4\%). The most prevalent age group was between $45-49$ 
years (30.5\%). Of respondents classified as non-smokers (33.3\%) most live with smokers in the workplace (51.4\%). Although the prevalence was lower than other studies conducted in Brazil, we suggest the development of measures to raise awareness about the practice aimed at tobacco prevention of smoking in this population.

Key-words: smoking, employees, prevalence, characteristics.

\section{Resumen}

\section{El hábito de fumar entre los empleados de una institución de educación superior}

El tabaquismo es una preocupación importante en la sociedad moderna debido a los efectos nocivos para la salud de la población. El conocimiento de la prevalencia de tabaquismo es necesario para realizar programas institucionales adecuados cuyo objetivo sea reducir el número de fumadores. Ante esta situación, el presente estudio tuvo como objetivo evaluar la prevalencia de tabaquismo entre los empleados de la Fundação Paulista de Tecnologia e Educaçáo (FPTE) Lins/SP. En este análisis, se administró un cuestionario a 149 empleados y se encontró una prevalencia de tabaquismo del 15,4\%. El $74,5 \%$ de los empleados no eran fumadores y el $10,1 \%$ eran ex fumadores. La prevalencia de tabaquismo por sexo fue de $65,2 \%$ en hombres y 34,8\% entre las mujeres. La mayoría llevaban fumando más de 9 años $(87,0 \%)$ y comenzaron a fumar entre los 16 y 18 años (43,4\%). El grupo de edad más frecuente fue entre 45-49 ańos (30,5\%). De los encuestados clasificados como no fumadores $(33,3 \%)$, la mayoría viven con fumadores en el puesto de trabajo $(51,4 \%)$. Aunque la prevalencia fue menor que en otros estudios realizados en Brasil, se sugiere la elaboración de medidas para aumentar la concienciación acerca de la práctica de fumar dirigida a la prevención del tabaquismo en esta población.

Palabras-clave: tabaquismo, empleados, prevalencia, características.

\section{Introdução}

O tabagismo é considerado o mais importante problema de saúde pública e a principal causa evitável de morte nos dias atuais. A cada ano, aproximadamente cinco milhóes de pessoas morrem por doenças relacionadas ao tabaco e a previsão é de que, persistindo o atual modelo de consumo, em 2030, serão oito milhões de mortes ao ano, das quais mais de $80 \%$ ocorrerão nos países em desenvolvimento [1].

A literatura científica atual, além de estimar o risco atribuível ao fumo ativo e passivo associado a várias doenças, mostra que a cessação do tabagismo oferece benefício mesmo entre os mais idosos [2].

Em nosso país, o tabagismo está diretamente relacionado a $30 \%$ dos infartos do miocárdio, 25\% dos acidentes vasculares cerebrais, $85 \%$ das mortes por câncer de pulmão, sendo o fator isolado causal de $30 \%$ da mortalidade total [3]. Acredita-se que, se não forem tomadas medidas adequadas para o controle dessa pandemia, próximo ao ano de 2020 o tabagismo será o responsável por 10 milhóes de mortes por ano, com proporçôes de uma em cada seis pessoas consumidoras de tabaco.

Sabe-se que o Brasil é o maior exportador de folhas de tabaco e o quarto produtor de fumo no mundo, sendo que as maiores plantaçóes de fumo do país estão no Rio Grande do Sul. Nos últimos 30 anos, foram consumidos internamente quase três milhôes de toneladas de folhas para a fabricação de 3,5 trilhóes de cigarros. Com essa produção, foram liberadas, no meio ambiente, 50 mil toneladas de alcatrão, 50 mil toneladas de monóxido de carbono e 50 toneladas de amônia [4].

Este panorama, portanto, representa um sério problema de saúde pública para qualquer país, independentemente do seu grau de desenvolvimento. É extremamente útil a atualizaçáo dos estudos referentes ao tabaco, pois o mesmo náo atinge com seus malefícios apenas seus usuários, mas também pessoas próximas aos mesmos. É importante ressaltar que o fumante muitas vezes se encontra ao lado de crianças tornando-as fumantes passivos [5].

Portanto, o presente estudo teve como objetivos identificar a prevalência do hábito de fumar e a ocorrência de fatores associados ao tabagismo em funcionários da Fundação Paulista de Tecnologia e Educação (FPTE) de Lins/SP, além de desenvolver estratégicas que visem a profilaxia do consumo de cigarros nesta populaçáo.

\section{Material e métodos}

Realizou-se um estudo transversal com os funcionários da FPTE. Para se calcular o tamanho 
da amostra, utilizou-se a população total de 425 funcionários e erro amostral tolerável de $5 \%$ para possibilitar intervalo de confiança de 95\%. Dessa maneira, foi obtida, inicialmente, como amostra ideal para o desenvolvimento deste estudo, um grupo de 149 indivíduos [6].

Para obtenção das informações acerca do consumo de tabaco, utilizou-se um questionário pré-codificado, individual e confidencial, com perguntas relacionadas ao hábito tabágico, distribuídas em três domínios, direcionadas aos fumantes regulares e fumantes ocasionais, aos ex-fumantes e aos não fumantes. Este questionário continha variáveis demográficas (idade, sexo e cor), sócio-econômica (renda familiar em salários mínimos vigentes na época da pesquisa), variáveis relacionadas ao tabagismo (se é fumante, tipo de fumo, consumo diário, idade de início, tempo de tabagismo e, caso ex-fumante, tempo de tabagismo e de suspensáo do fumo), tipo de tabaco (industrializado, confeccionado pelo próprio fumante, charuto e cachimbo), convivência com fumantes (pais, irmãos, amigos), local de convivência (casa, universidade, trabalho). Também foram investigados o consumo de bebidas alcoólicas, prática de esportes e presença de sintomas como tosse seca, tosse produtiva e sibilância.

O ponto de corte para a definição de fumante foi "ter fumado um ou mais cigarros por dia há pelo menos um mês", e para ex-fumantes "aqueles que na ocasiáo da pesquisa não eram fumantes, mas que o haviam sido no passado". As perguntas sobre sintomas respiratórios foram referentes aos últimos doze meses, excluindo-se os sintomas ocorridos durante gripes.

O questionário foi aplicado diretamente aos funcionários após esclarecimentos e orientaçôes básicas sobre os objetivos da pesquisa em questão, ficando o indivíduo livre para decidir sobre sua participação. Em se fazendo a opção por participar da pesquisa, o mesmo assinou o "Termo de Consentimento Livre e Esclarecido (TCLE)". O trabalho foi aprovado pelo Comitê de Ética em Pesquisa com Seres Humanos do Centro Universitário de Lins/SP - Unilins, sob o protocolo $01 / 2011$.

Foi utilizado o teste do qui-quadrado para avaliar a existência de associaçóes entre as variáveis analisadas, sendo considerado o valor de $\mathrm{p}<0,05$ para significância estatística.

\section{Resultados e discussão}

O questionário auto-respondido apresentou diversas vantagens, que se referem não só à diminuição de custos e do tempo necessário para desenvolver a pesquisa, bem como mostrou-se ideal naquele ambiente de trabalho, já que os funcionários responderam durante o expediente, no momento mais disponível, sem prejuízos das suas funçôes. Por outro lado, o questionário respondido por eles próprios, sem prejuízo em suas funções na empresa e com a garantia do sigilo das informaçóes, possivelmente incentivou respostas mais sinceras e evitou constrangimentos quanto a informar problemas de saúde e hábitos socialmente indesejáveis. Assim, provavelmente, esses fatores contribuíram também para a grande adesáo à pesquisa.

O tabagismo é considerado o principal fator de risco passível de prevenção para várias doenças crônicas e é responsável por um grande número de mortes prematuras no mundo [7].

Nesta pesquisa, do total de entrevistados (149), $49,7 \%$ eram do sexo masculino (74) e 50,3\% do feminino (75), portanto, não foi encontrada diferença estatisticamente significativa $(p>0,05)$. Foi observado que $15,4 \%$ dos funcionários apresentaram-se como fumantes, $10,1 \%$ foram classificados como ex-fumantes e 74,5\% não eram fumantes (Tabela I).

Tabela I - Prevalência total de tabagismo entre os funcionários da FPTE.

\begin{tabular}{lcc}
\hline \multicolumn{1}{c}{ Uso do Tabaco } & $\mathbf{n}$ & $\%$ \\
\hline Fumantes & 23 & 15,4 \\
Ex-fumantes & 15 & 10,1 \\
Não fumantes & 111 & 74,5 \\
Total & 149 & 100,0 \\
\hline
\end{tabular}

Considerando apenas os tabagistas (23), encontramos uma prevalência de 65,2\% (15) no sexo masculino, enquanto que no sexo feminino a mesma foi de 34,8\% (8) (Figura 1), contrariando a tendência mundial de aproximação da prevalência de fumantes entre os sexos, através de seu declínio entre os homens e do aumento ou estabilização entre mulheres [8]. Todos os fumantes deste estudo declararam que utilizam cigarros comercializados.

A prevalência de fumantes do sexo masculino $(19,7 \%)$ foi inferior às estimativas de outras pesquisas nacionais, assim como a proporçáo de mulheres $(11,0 \%)$ (Tabela II). Pesquisas realiza- 
das com trabalhadores de uma empresa bancária revelaram $31,1 \%$ de tabagismo entre os homens, enquanto que a incidência entre as mulheres foi de 27,8\% [8]. Estudos anteriores, na regiáo de Porto Alegre, encontraram uma prevalência de fumantes ao redor de $41,5 \%$ entre os homens e $29,5 \%$ entre as mulheres [3]. Em doze capitais brasileiras, as prevalências foram $45,0 \%$ entre os homens e 33,0\% entre as mulheres [7].

Figura 1 - Estratificação da amostra segundo o sexo entre os tabagistas.

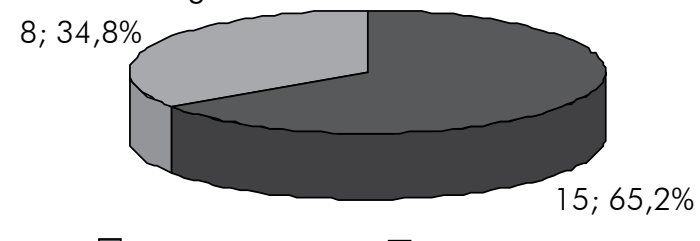

Homens $\quad \square$ Mulheres

Tabela II - Prevalência de taba gismo segundo o sexo na população estudada.

\begin{tabular}{lcccc}
\hline \multirow{2}{*}{ Uso do Tabaco } & \multicolumn{2}{c}{ Homens } & \multicolumn{2}{c}{ Mulheres } \\
\cline { 2 - 5 } & $\mathbf{n}$ & $\%$ & $\mathbf{n}$ & $\%$ \\
\hline Fumantes & 15 & 19,7 & 8 & 11,0 \\
Ex-fumantes & 9 & 11,9 & 6 & 8,2 \\
Não-fumantes & 52 & 72,4 & 59 & 80,8 \\
\hline Total & 76 & 100,0 & 73 & 100,0 \\
\hline
\end{tabular}

A caracterização do tabagismo entre os funcionários da FPTE está apresentada na Tabela III. Nota-se que a maioria dos indivíduos fumantes (7) consome entre 16 a 20 cigarros diariamente (30,0\%).

A maioria $(43,4 \%)$ iniciou-se na prática quando tinha entre 16 a 18 anos de idade. Também entre os funcionários da empresa estudada o hábito de fumar iniciou-se principalmente na adolescência, conforme já descrito em outros estudos [3,9,10], reforçando a importância de estratégias de controle de propagandas e marketing sobre o cigarro dirigidas a esta faixa etária. E, por fim, observa-se a predominância de 87,0\% das pessoas tabagistas com mais de 9 anos na prática.

Dos motivos que levaram os tabagistas ao início da prática, observou-se o predomínio dos itens "influência dos amigos" e "vontade própria" com $56,5 \%$ e 26,2\%, respectivamente. Em menores proporçôes "influência dos pais" com $8,7 \%$ e "efeito da propaganda e modismo" com $4,3 \%$ cada um (Tabela IV).
Tabela III - Características do tabagismo entre os funcionários da FPTE quanto ao número de cigarros fumados por dia, idade de início do tabagismo e tempo de fumo.

\begin{tabular}{|c|c|c|}
\hline & $\mathrm{n}$ & $\%$ \\
\hline \multicolumn{3}{|c|}{ Número de cigarros fumados por dia } \\
\hline 1 a 3 & 3 & 13,0 \\
\hline 4 a 6 & 2 & 8,7 \\
\hline 7 a 9 & 1 & 4,3 \\
\hline 10 a 12 & 5 & 21,4 \\
\hline 13 a 15 & 1 & 4,3 \\
\hline 16 a 20 & 7 & 30,0 \\
\hline$>20$ & 4 & 17,3 \\
\hline \multicolumn{3}{|c|}{ Idade de início no fumo (anos) } \\
\hline$<12$ & 2 & 8,7 \\
\hline 12 a 15 & 3 & 13,0 \\
\hline 16 a 18 & 10 & 43,4 \\
\hline 19 a 21 & 6 & 26,2 \\
\hline 22 a 24 & - & - \\
\hline$>24$ & 2 & 8,7 \\
\hline \multicolumn{3}{|c|}{ Tempo de fumo (anos) } \\
\hline 1 a 3 & - & - \\
\hline 4 a 6 & 1 & 4,3 \\
\hline 7 a 9 & 2 & 8,7 \\
\hline$>9$ & 20 & 87,0 \\
\hline
\end{tabular}

Tabela IV - Motivos pelos quais os tabagistas iniciaram-se na prática.

\begin{tabular}{lcc}
\hline \multicolumn{1}{c}{ Motivo } & $\mathbf{n}$ & $\%$ \\
\hline Influência dos Amigos & 13 & 56,5 \\
Vontade Própria & 6 & 26,2 \\
Influência dos Pais & 2 & 8,7 \\
Efeito da Propaganda & 1 & 4,3 \\
Modismo & 1 & 4,3 \\
Total & 23 & 100,0 \\
\hline
\end{tabular}

Em relação aos fumantes, quando questionados sobre o aconselhamento de profissionais da saúde para a cessação da prática tabágica explicando os malefícios desta, $86,4 \%$ declararam que sim. Já $13,6 \%$ nunca tiveram recomendaçóes dos profissionais deste seguimento. A elevada prevalência de fumantes interessados em deixar o vício $(77,3 \%)$ sugere que, neste grupo, açóes para a cessação do tabagismo seriam eficazes e, possivelmente, poderiam trazer efeitos amplamente positivos [5,11].

Verificou-se que $54,5 \%$ dos tabagistas fumam mesmo estando doente, enquanto a maioria pretende deixar de fumar $(77,3 \%)$, sendo que, quando questionados sobre sua dependência ao vício, $86,4 \%$ subestima a mesma dizendo que é capaz de deixar a prática. Outro ponto que merece destaque é que $68,2 \%$ dos tabagistas relatam que o cigarro lhe faz mal, entretanto, continuam fumando (Tabela V). 
Tabela V - Perfil dos indivíduos tabagistas da FPTE.

\begin{tabular}{lcc}
\hline & Sim (\%) & Não (\%) \\
\hline Fuma mesmo estando doente & 54,5 & 45,5 \\
Pretende deixar de fumar & 77,3 & 22,7 \\
Acredita que é capaz de deixar & 86,4 & 13,6 \\
o vício & 22,7 & 77,3 \\
Já tentou parar de fumar & 68,2 & 31,8 \\
\hline O cigarro lhe faz mal & & \\
\hline
\end{tabular}

Com relação aos indivíduos que se declararam ex-fumantes (15), os motivos principais citados para a interrupção deste hábito foram: força de vontade $(88,2 \%)$, conscientização dos malefícios do tabagismo $(5,9 \%)$ e problemas de saúde $(5,9 \%)$.

Dos entrevistados classificados como não-fumantes (111), 33,3\% da amostra (37) convive com fumantes, sendo esta convivência, em sua maioria, no ambiente de trabalho $(51,4 \%)$ (Figura 2).

Figura 2 - Distribuição dos indivíduos não fumantes de acordo com a convivência com fumantes.

$5 ; 13,5 \%$

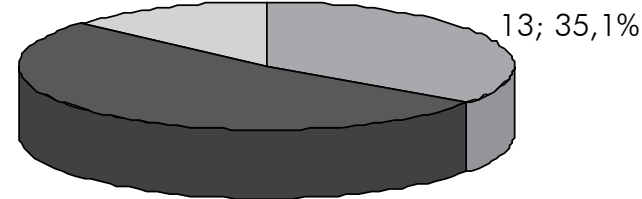

$19 ; 51,4 \%$

$\square$ Domicílio $\square$ Trabalho $\square$ Outros

\section{Conclusão}

Nesta pesquisa observou-se a prevalência total de $15,4 \%$ de tabagismo entre os funcionários da FPTE. Embora este índice esteja abaixo quando comparado a outras pesquisas do gênero, sugere-se a elaboração de medidas de conscientização sobre a prática tabágica visando à profilaxia do consumo de cigarros nesta população. Uma das estratégias, frequentemente utilizadas para alcançar este objetivo, tem sido a implantação de programas de promoção da saúde nas empresas. $\mathrm{O}$ ambiente de trabalho tem sido apontado como um local favorável para modificar hábitos, já que neste, os funcionários passam a maior parte do dia, proporcionando excelentes oportunidades para açóes educativas, o que permite reduzir assim, o número de absenteísmo e gastos com doenças. A maioria das empresas de países desenvolvidos criou legislação específica proibindo ou restringindo o uso de cigarros em locais coletivos. Estas medidas constituem conquistas importantes para os não-fumantes e têm estimulado o abandono ao hábito de fumar, bem como a redução do número de cigarros fumados entre os trabalhadores.

\section{Referências}

1. Hallal ALC, Gotlieb SLD, Almeida LM, Casado L. Prevalência e fatores associados ao tabagismo em escolares da Regiáo Sul do Brasil. Rev Saúde Pública 2009;43(5):779-88.

2. World Health Organization. Summary: surveillance of risk factors for noncommunicable diseases. The $\mathrm{WHO}$ STEP wise approach. Geneva: WHO; 2001.

3. Moreira LB, Fuchs FD, Moraes RS, Bredemeir M, Cardozo S. Prevalência de tabagismo e fatores associados em área metropolitana da região Sul do Brasil. Rev Saúde Pública 1995;29(1):46-51.

4. Achutti A, Menezes AMB. Epidemiologia do tabagismo. In: Guia Nacional de Prevençáo e Tratamento do Tabagismo. Rio de Janeiro: Vitrô Comunicação; 2001. p. 9-27.

5. Miranda JS, Almeida JB, Marques SFG. Prevalência e fatores associados ao tabagismo em uma unidade universitária. Enfermagem Brasil 2009;8(5):266-71.

6. Oliveira EFT, Grácio MCC. Análise a respeito do tamanho de amostras aleatórias simples: uma aplicação na área de Ciência da Informação. Rev Ciência Informação 2005;6(3).

7. World Health Organization. The world health report 2002. Reducing risks, promoting healthy life. Geneva: WHO; 2002.

8. Ministério da Saúde, Secretaria de Assistência à Saúde. Doenças Cardiovasculares no Brasil. SUS. 1a ed. Brasília: Coordenação de Doenças Cardiovasculares; 1993. 36p.

9. Griep RH, Chór D, Camacho LAB. Tabagismo entre trabalhadores de empresa bancária. Rev Saúde Pública 1998;32(6):533-40.

10. Elders MJ, Perry CL, Eriksen MP, Giovino GA. The Report of the Surgeon General: preventing tobacco use among young people. Am J Public Health 1994;84:543-7.

11. Rodrigues ESR, Cheik NC, Mayer AF. Nível de atividade física e tabagismo em universitários. Rev Saúde Pública 2008;2(4):672-8. 